\title{
Ruolo del citrato nel metabolismo osseo
}

\author{
Giuseppe Vezzoli, Giulia Magni, Monica Avino, Teresa Arcidiacono
}

Unità di Nefrologia e Dialisi, IRCCS Ospedale San Raffaele, Scuola di Specializzazione in Nefrologia, Università Vita Salute San Raffaele, Milano - Italia

\begin{abstract}
Role of Citrate in Bone Metabolism
Citrate is an organic compound involved in tricarboxylic acid cycle, regulation of acid-base balance, lipid metabolism and bone formation. The $90 \%$ of body citrate is deposited in bone tissue and is released with calcium ions during bone resorption; therefore, bone resorption contributes to maintain normal plasma levels of citrate together with kidney excretion. The parallel release of citrate and calcium from bones decreases the possibility of calcium-phosphate precipitation in soft tissues, as citrate can bind calcium ions in organic fluids. Citrate may also take part to the bone formation as it sustains the correct mineralization of bone organic matrix: its molecule binds calcium ions at the surface of hydroxyapatite nanocrystals and maintains the correct spatial disposition of nanocrystals, thus, stabilizing the structure of bone lamellae and sustaining biomechanical characteristics of bone tissue. Multiple studies observed that citrate administration significantly increased areal and volumetric bone mineral density at different locations of $1-2 \%$ per year and improved bone resorption markers as well. Therefore, it has been hypothesised a therapeutic role of citrate in osteoporosis; however, this role has to be better clarified to understand its real anti-fracture effect.
\end{abstract}

Keywords: Bone remodeling, Citrate, Citrate excretion, Hypocitraturia

\section{Introduzione}

Il citrato è un composto organico che è coinvolto in fondamentali processi metabolici nell'organismo umano. II principale di questi è la produzione dei substrati energetici che avviene attraverso il ciclo degli acidi tricarbossilici del quale il citrato è un metabolita intermedio (Fig. 1). II citrato è anche necessario per la regolazione del bilancio acido-base, il metabolismo lipidico e la formazione ossea (1-4). Dal punto di vista molecolare, il citrato è un acido organico tricarbossilico con peso molecolare di $189 \mathrm{D}$. I gruppi carbossilici della sua molecola hanno rispettivamente un pK di 3,13, 4,76 e 6,4; perciò il $95 \%$ del citrato è presente nel sangue come tricarbossilato, il $4 \%$ come dicarbossilato e l' $1 \%$ come monocarbossilato (5). Il citrato circola nel torrente ematico libero da legame con le

Received: December 20, 2019

Accepted: December 31, 2019

Published online: February 20, 2020

Indirizzo per la corrispondenza:

Giuseppe Vezzoli MD

Unità di Nefrologia e Dialisi

Istituto Scientifico Universitario San Raffaele

Via Olgettina 60

20142 Milano, Italy

vezzoli.giuseppe@hsr.it proteine plasmatiche ed è quindi completamente disponibile per la filtrazione glomerulare. Circa il $75 \%$ del citrato filtrato viene riassorbito nel tubulo prossimale (Fig. 2) ed il $25 \%$ è escreto con le urine (3). Nel sangue circola però solo una piccola quota del citrato corporeo, perché il $90 \%$ è depositato nel tessuto osseo che rappresenta perciò l'organo dove viene conservato (6). Durante il riassorbimento il citrato viene rilasciato dallo scheletro e per questo motivo il metabolismo osseo contribuisce a mantenere i normali livelli plasmatici di citrato insieme all'escrezione renale. L'apporto alimentare ha invece scarsa influenza sui livelli plasmatici del citrato, anche se ogni giorno ne vengono ingeriti circa 4 grammi (21 mmoli) attraverso il consumo dei vegetali, soprattutto gli agrumi (7).

È noto come il citrato interagisca con gli ioni calcio nelle urine e nei tessuti molli per proteggere dalla precipitazione dei sali di calcio nei tessuti. In questo ambito, il citrato rappresenta un composto di estrema importanza anche per il tessuto osseo; esso viene infatti depositato tra i cristalli di apatite durante il processo di formazione ossea con lo scopo di mantenere il normale orientamento tridimensionale dei nanocristalli di apatite nelle lamelle ossee. Questa modalità di deposizione garantisce che il tessuto osseo sviluppi le sue tipiche proprietà biomeccaniche (8). Questo articolo illustra il ruolo del citrato nella formazione del tessuto osseo e le modalità di interazione del citrato con le altre componenti minerali del tessuto osseo. 


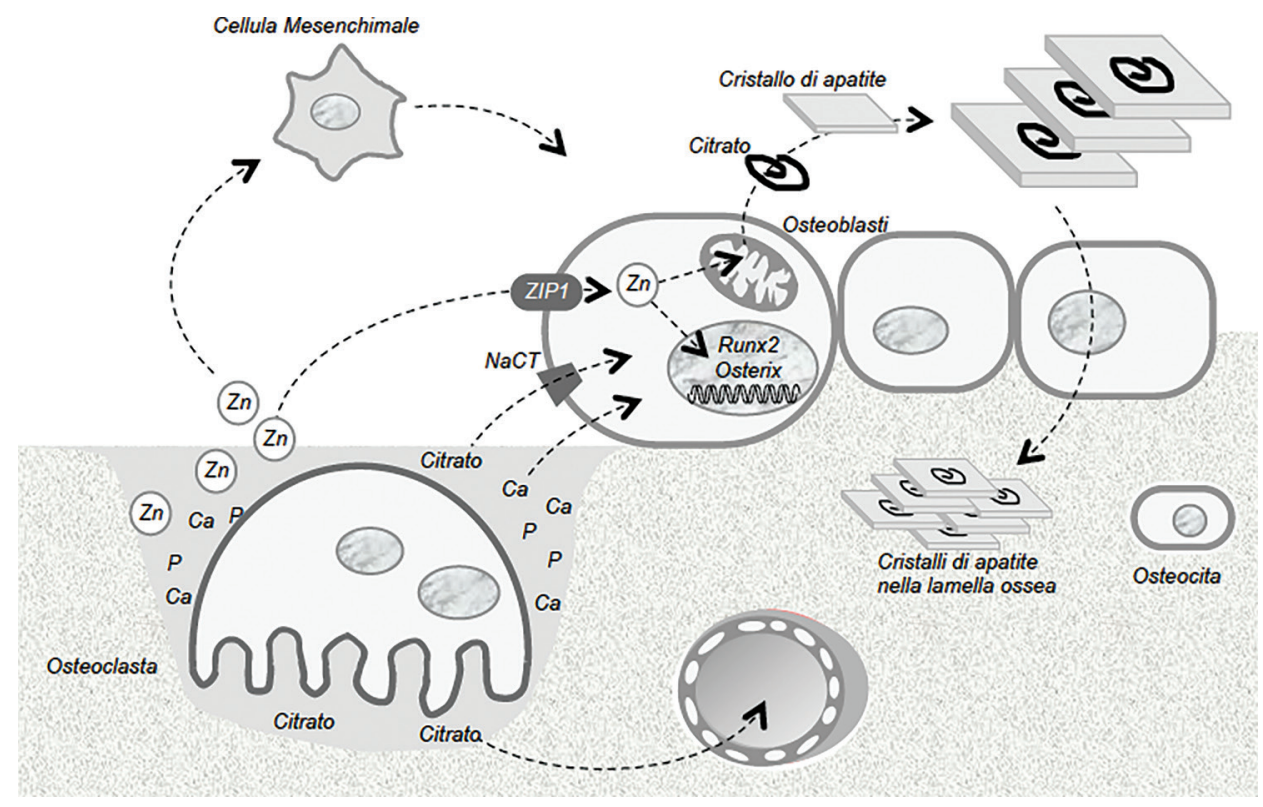

Fig. 1 - Schema del ciclo degli acidi tricarbossilici. Questo processo avviene nei mitocondri e genera energia attraverso la fosforilazione del GDP a GTP e la formazione di fattori riducenti NADH e $\mathrm{FADH}_{2}$, che saranno poi utilizzati per la produzione di ATP; inoltre esso mette a disposizione della cellula diversi precursori utilizzabili nella gluconeogenesi, nella sintesi degli acidi grassi, del colesterolo e nella biosintesi dei nucleotidi.

\section{Citrato e rimodellamento osseo}

Il citrato rappresenta il $5 \%$ della componente organica del tessuto osseo e I'1-2\% del tessuto osseo totale. La sua concentrazione nello scheletro è 5-20 $\mu \mathrm{mol} /$ grammo, mentre nei tessuti molli la concentrazione è <1 $\mu \mathrm{mol} /$ grammo, tranne nella prostata, dove sale a circa $10 \mu \mathrm{mol} /$ grammo. Nello scheletro il citrato viene sottoposto ad un continuo ricambio che segue le fasi del rimodellamento osseo (9): esso viene perciò rimosso nel corso del riassorbimento e depositato nello scheletro dagli osteoblasti durante la deposizione di nuovo tessuto. II citrato rilasciato dal tessuto osseo durante il riassorbimento viene riversato nel sangue e determina la concentrazione del citrato ematico. Viceversa, nel processo di costruzione delle lamelle ossee gli osteoblasti utilizzano il citrato che viene generato dal metabolismo cellulare (10-12). Il bilancio tra la quota di citrato rilasciata dallo scheletro e quella che viene usata per la sua costituzione dipende dalla disponibilità di citrato e dalle condizioni del rimodellamento osseo ed è possibile che il pool scheletrico di citrato diminuisca nel corso della vita soprattutto nei soggetti osteoporotici (13).

Oltre al citrato, il riassorbimento osseo provoca la liberazione di citochine, fattori di crescita (TGF $\beta$, IGF), proteine ossee morfogenetiche (BMP) e ioni calcio e zinco $(14,15)$. Queste sostanze stimolano l'attività osteosintetica degli osteoblasti e tra esse lo zinco sembra regolare la disponibilità di citrato per l'organizzazione tridimensionale delle lamelle ossee (Fig. 3) (12). Gli ioni zinco stimolano la differenzia- zione in senso osteoblastico delle cellule mesenchimali e la produzione dei fattori di trascrizione osteogenetici Runx2 ed Osterix che a loro volta attivano l'espressione della proteina ZIP-1 sulla membrana plasmatica di queste cellule (12). La proteina ZIP-1 si comporta come un carrier che media l'influsso dello zinco negli osteoblasti; lo ione zinco nel citoplasma attiva la citrato-sintasi e la conseguente produzione di citrato che diventa così disponibile per essere depositato nelle lamelle del nuovo tessuto osseo (12). Il processo di deposizione prevede che almeno due dei residui carbossilati della molecola del citrato siano legati a ioni calcio prossimi alla superficie dei nanocristalli di idrossiapatite; il citrato risulta così inserito tra i nanocristalli secondo una disposizione che stabilizza la struttura delle lamelle ossee $(16,17)$. È stato calcolato che i gruppi carbossilici del citrato distino 0,3-0,45 nm dalla superficie dei nanocristalli e che il citrato copra l'idrossiapatite con una densità pari ad una molecola ogni $2 \mathrm{~nm}^{2}$. Questa particolare interazione strutturale tra citrato ed apatite rende i nanocristalli meno idrofili e fa in modo che la dimensione dei cristalli di idrossiapatite non superi i $3 \mathrm{~nm}$, una misura ritrovata in diversi vertebrati e che può essere considerata come ottimale per garantire le proprietà biomeccaniche dell'osso in termini di resistenza e resilienza (16-18). È verosimile che gli osteoblasti, oltre ad utilizzare il citrato da essi prodotto, possano riutilizzare il citrato interstiziale osseo sfruttando il lavoro dei carrier di membrana che trasportano il citrato nel loro citoplasma. A tale riguardo è stato osservato che i topi knockout per SLC13A5, codificante il carrier d'influsso $\mathrm{NaCT}$ (Fig. 2), sviluppano una ridotta massa 


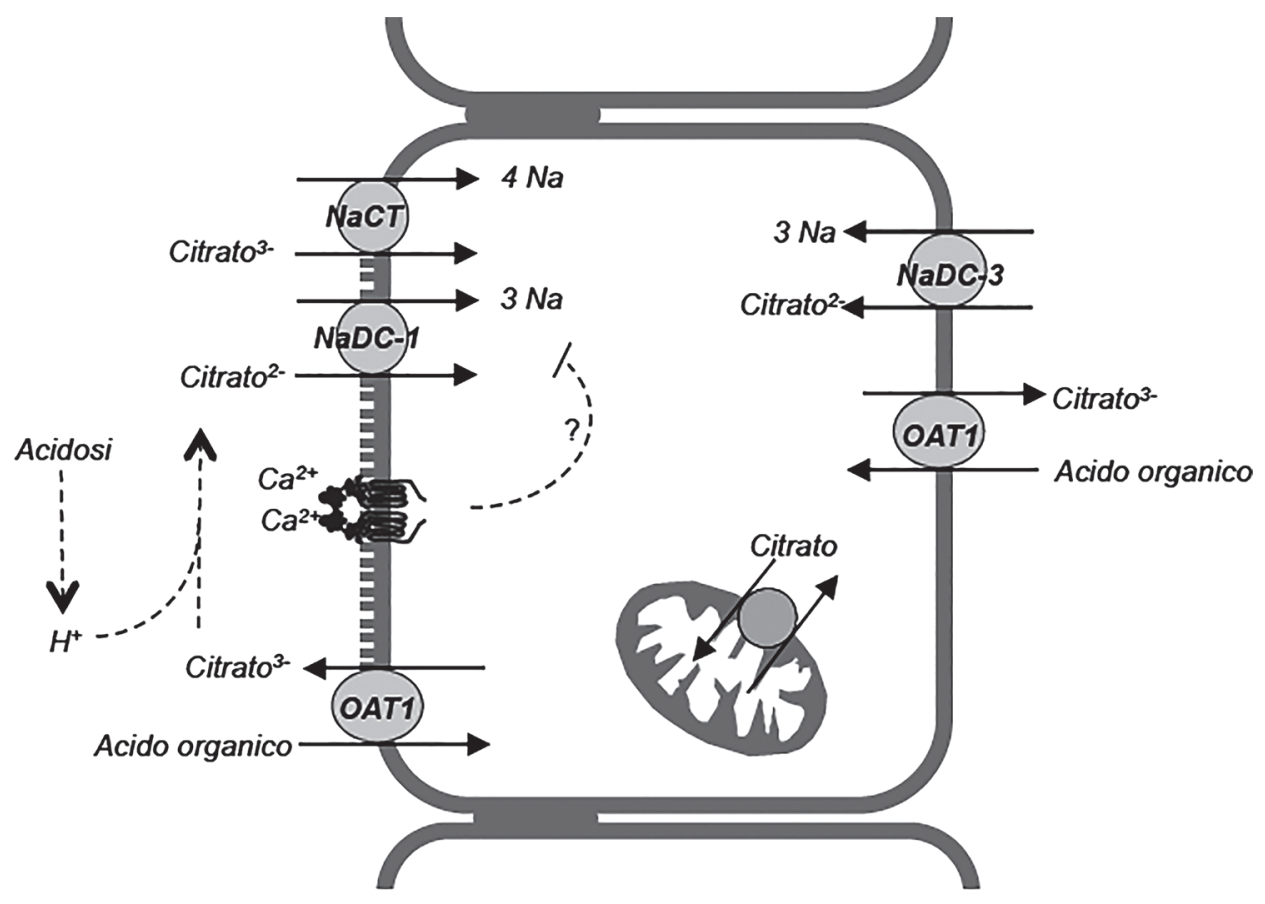

lume

interstizio

Fig. 2 - La membrana plasmatica della cellula tubulare prossimale possiede diversi carrier per trasporto del citrato e degli altri dicarbossilati (malato, chetoglutarato, succinato etc). Il carrier NaCT (SLC13A5, crom 17p13) trasporta nella cellula una molecola di citrato, come tri- o dicarbossilato, insieme a 4 ioni sodio. La membrana cellulare possiede anche due carrier NaDC (NaDC1 e NaDC3) che trasportano citrato nella cellula insieme a 3 ioni sodio. NaDC1 (SLC13A2, crom 17p13.2) è un carrier a bassa affinità, espresso sulla membrana apicale delle cellule tubulari prossimali e responsabile del riassorbimento renale del citrato e degli altri acidi dicarbossilici. NaDC3 (SLC13A3, crom 20q13.12) è un carrier ad alta affinità espresso sulla membrana basolaterale del tubulo prossimale ed è coinvolto nella escrezione degli acidi organici e nella eliminazione di farmaci o sostanze tossiche. OAT1 (SLC22A6, crom 11q12.3) è un carrier espresso sia sulla membrana apicale che basolaterale delle cellule tubulari prossimali che scambia un anione con un dicarbossilato; OAT1 secerne nel lume tubulare metaboliti endogeni, come i dicarbossilati, o esogeni come le sostanze tossiche o i farmaci.

II riassorbimento del citrato sembra essere inibito dalla attivazione del recettore sensibile al calcio (CaSR) da parte degli ioni calcio presenti nel lume tubulare. II meccanismo di questo effetto non è stato ancora chiarito e sembra dedicato ad accrescere l'escrezione di citrato nel caso di ipercalcemia.

Nei mitocondri, tre carrier consentono al citrato e agli altri dicarbossilati di attraversare la membrana mitocondriale. II CTP (SLC25A1, crom 22q11.21) scambia citrato con un altro dicarbossilato ed è espresso nel rene; DIC (SLC25A10, crom 17q25.3) è stato trovato nei mitocondri di rene e scambia un dicarbossilato con uno ione fosfato, solfato o tiosolfato oppure con un altro dicarbossilato; OGC (oxoglutarate carrier; SLC25A11, crom 17p13.2), è uno scambiatore elettroneutro malato/dicarbossilato che è stato ritrovato nel rene.

ossea femorale mentre lo smalto nei denti è assente alla tredicesima settimana di vita (19).

\section{Citrato e massa minerale ossea}

Diversi studi nell'animale e nell'uomo hanno dimostrato come la quantità e la qualità del tessuto osseo dipendano dalla disponibilità del citrato nell'organismo. Ceppi di ratto che sviluppavano osteoporosi secondaria a cause come l'invecchiamento, l'ovariectomia e la somministrazione di acido retinoico, hanno manifestato una riduzione della concentrazione ematica di citrato e del contenuto di citrato nel tessuto osseo sia corticale che trabecolare (13). Un recente studio trasversale di coorte che includeva 87 soggetti normali (25 maschi and 62 femmine) ha osservato come la concentrazione plasmatica del citrato fosse correlata positivamente con la densità minerale ossea lombare e femorale (13). Un altro studio che considerava un campione di 60 donne sane ha rilevato che l'escrezione del citrato fosse positivamente correlata con la densità minerale ossea radiale e con la concentrazione ematica della vitamina $D$, mentre era negativamente correlata con i valori del PTH plasmatico (20).

Oltre agli studi trasversali, anche gli studi di intervento hanno dimostrato che la somministrazione di citrato di potassio aveva un effetto positivo sulla mineralizzazione ossea. In uno di questi studi, il citrato di potassio è stato somministrato per os ( $60 \mathrm{mEq}$ die) a 91 soggetti sani, che sono stati confrontati con 90 soggetti sani ai quali è stato somministrato placebo; in entrambi i gruppi i partecipanti avevano età maggiore di 65 anni, non erano affetti da osteoporosi ed assumevano vitamina $\mathrm{D}$ e calcio oltre al citrato o al placebo (21). Nel corso di due anni di follow-up, la 


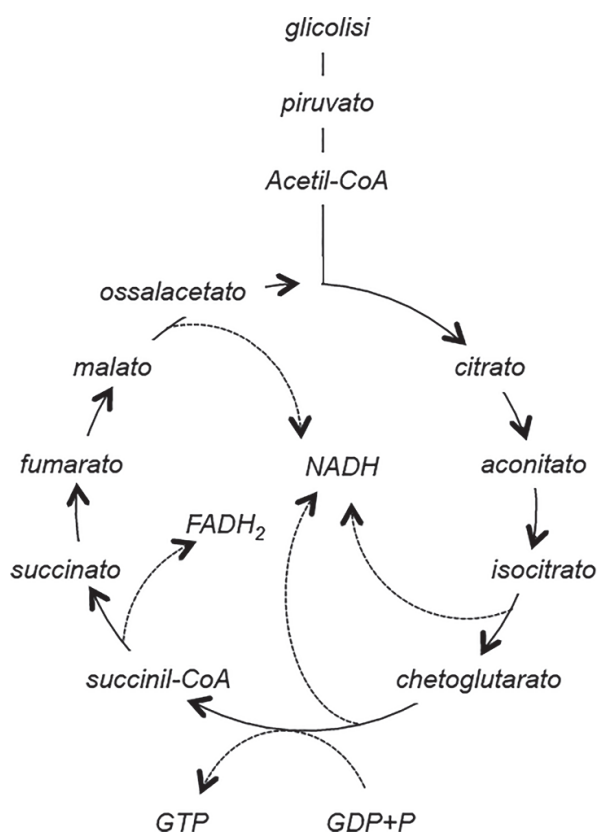

Fig. 3 - La formazione del tessuto osseo prevede la formazione dei cristalli di idrossipatite e la loro organizzazione in lamelle. Affinché il tessuto osseo possa sviluppare le tipiche caratteristiche biomeccaniche, i cristalli devono essere disposti secondo una architettura nella quale la molecola del citrato viene inserita tra i nanocristalli. Gli osteoblasti sono le cellule deputate a questa organizzazione e lo ione zinco è fondamentale per stimolare gli osteoblasti a produrre citrato per la osteosintesi.

BMD sia areale che volumetrica ha presentato un significativo aumento: la BMD areale misurata a livello femorale e lombare con la DXA era aumentata mediamente dell' $1,7 \%$; la BMD volumetrica esplorata con la pQCT era aumentata dell' $1,3 \%$ in sede radiale e del $2 \%$ in sede tibiale; tuttavia, solo la componente trabecolare si mostrava significativamente aumentata e coinvolgeva nell'aumento sia il numero che il volume delle trabecole, a dimostrazione di un miglioramento della microarchitettura ossea (21). L'incremento dei valori di densità minerale ossea radiale era osservato anche in una casistica italiana di 109 pazienti con calcoli renali (51 uomini e 58 donne) trattati per due anni con 40 $\mathrm{mEq}$ di citrato di potassio (22) ed in uno studio americano su 161 donne in post-menopausa trattate con $30 \mathrm{mEq}$ di citrato di potassio per un anno (23).

Anche i marcatori di riassorbimento osseo riducevano i loro valori dopo somministrazione di citrato. Uno studio su 22 donne osteopeniche italiane ha dimostrato come la idrossiprolina e la deossipiridinolina urinarie diminuivano significativamente di circa il $30 \%$ dopo 3 mesi di trattamento con citrato di potassio $(0,8-1 \mathrm{~g} / \mathrm{kg}$ die) (24). Uno studio su 83 donne americane affette da osteopenia postmenopausale ha osservato una diminuzione dell'escrezione urinaria di telopeptide $\mathrm{N}$-terminale del collagene tipo I e dei livelli ematici del peptide $\mathrm{N}$-terminale del procollagene tipo I dopo un anno di trattamento con $40 \mathrm{mEq}$ di citrato di potassio. Lo studio concludeva perciò che il turnover osseo era significativamente ridotto dalla terapia con citrato (25).

Effetti positivi sulla massa minerale ossea sono stati osservati anche dopo somministrazione di sostanze alcaline diverse dal citrato, come il bicarbonato di potassio o sodio. L'effetto osteogenetico del citrato sembra perciò spiegato dall'aumento del carico di alcali che potrebbe ridurre l'efflusso di calcio dall'osso e l'escrezione di calcio attraverso una maggiore disponibilità di valenze alcaline nell'organismo ed un più stretto controllo del pH ematico e della concentrazione ematica del bicarbonato $(23,26)$.

\section{Influenza del metabolismo osseo sulla citratemia}

In condizioni basali, i livelli plasmatici del citrato sono mantenuti tra 19 e $29 \mathrm{mg} / \mathrm{l}(100-150 \mu \mathrm{mol} / \mathrm{l})$ come risultato dell'escrezione renale e del riassorbimento osseo $(27,28)$. Un marcato riassorbimento osseo, come nei casi delle osteopatie ad elevato turnover, rilascia ioni calcio e citrato nel fluido extracellulare e contribuisce a mantenere normale la concentrazione plasmatica di calcio e citrato. Gli ormoni che sostengono il riassorbimento osseo, come il PTH e la $1,25(\mathrm{OH})_{2}$ vitamina $\mathrm{D}$, aumentano perciò le concentrazioni plasmatiche di calcio e citrato; mentre per motivi opposti la calcitonina riduce la citratemia e la calcemia. Anche l'acidosi sistemica aumenta la concentrazione plasmatica di citrato perché stimola gli osteoclasti a riassorbire osso oltre che le cellule tubulari prossimali a recuperare il citrato filtrato $(27,29)$. Sembra quindi che esista un andamento parallelo tra il citrato e il calcio plasmatici che può avere lo scopo di ridurre la probabilità di precipitazione dei sali di calcio-fosfato (idrossiapatite o brushite) nei tessuti molli, dato che il citrato ha la capacità di legare gli ioni calcio nei fluidi organici rendendoli meno disponibili allo ione fosfato (o ossalato) ed alla formazione di sali poco solubili (30).

\section{Conclusioni}

Nella comune pratica clinica nefrologica, il citrato viene considerato come uno strumento diagnostico e terapeutico: viene infatti dosato nelle urine per esprimere il rischio di calcolosi renale e viene inoltre somministrato ai pazienti con calcolosi renale come sale di potassio e magnesio per prevenire le recidive. L'analisi della letteratura ha allargato questa prospettiva rivelando che il citrato interviene anche nella fisiopatologia osteo-scheletrica, un ambito che è stato fino ad oggi largamente ignorato. La variabile più interessante in ambito diagnostico sembra essere la citratemia, poiché essa viene influenzata dal riassorbimento osseo e potrebbe essere un marcatore del turnover osseo; purtroppo però, la citratemia non è stata studiata in modo sistematico nei pazienti con malattie ossee e per questo motivo non possiamo ancora attribuire ad essa un valore clinico e diagnostico specifico. 
Anche la citraturia potrebbe essere un marcatore del riassorbimento osseo, ma ancora una volta gli studi sono insufficienti per trarre delle conclusioni definitive.

Il campo della terapia sembra essere viceversa più promettente. Come è ben noto, diversi trials clinici di piccole dimensioni hanno osservato che il citrato di potassio e magnesio previene le recidive di calcolosi renale (31); oltre a queste osservazioni, diversi studi hanno utilizzato il citrato nella terapia dell'osteoporosi. L'effetto anti osteoporotico del citrato può essere legato al carico alcalino che consegue alla sua assunzione, ma potrebbe anche essere dovuto ad un effetto diretto sulla funzione osteoblastica e sull'anabolismo osseo. II citrato è infatti un composto fondamentale per la corretta organizzazione dei cristali di idrossiapatite nelle lamelle ossee, ma sembra anche avere un effetto diretto sugli osteoblasti che ne limita le attività osteolitiche (32). Le percentuali di incremento della massa minerale ossea ottenute dopo somministrazione del citrato sono intorno all'1,5\% per anno nelle diverse sedi ossee; perciò il citrato potrebbe essere un utile complemento alla terapia dell'osteoporosi in associazione ai farmaci anti riassorbitivi (denosumab, bisfosfonati) o anabolici (teriparatide, romosozumab). Potrebbe anche essere una utile terapia dell'osteopenia, quando i farmaci anti riassorbitivi possono essere giudicati eccessivi. Sulla base di queste considerazioni, da alcuni anni sono disponibili tra gli integratori alimentari dei preparati a base di citrato di sodio o potassio dedicati alla cura dell'osteoporosi. I pochi studi condotti hanno dimostrato che la posologia giornaliera del citrato dovrebbe essere di almeno $40 \mathrm{mEq} / \mathrm{die}(20 \mathrm{mmoli} /$ die, pari a circa 4 grammi di citrato di potassio) per ottenere una risposta clinica sostanziale. Tuttavia, gli studi sui benefici ossei del citrato hanno considerato target surrogati come la massa minerale e i marker di turnover osseo, perché non sono stati valutati fino ad oggi i possibili effetti sulle fratture incidenti. Nonostante questo grosso limite, l'effetto sulla massa ossea lascia intendere un potenziale effetto benefico del citrato sulle fratture.

In conclusione, possiamo perciò attribuire al citrato un ruolo terapeutico nella prevenzione della calcolosi renale e nella terapia dell'osteoporosi; il ruolo del citrato nel metabolismo osseo deve però essere ulteriormente chiarito per poter capire se possiede un reale effetto anti-fratturativo.

\section{Disclosures}

Financial support: Authors declare no financial support.

Conflict of interest: Authors declare no conflict of interest.

\section{Bibliografia}

1. Caudarella R, Vescini F, Buffa A, Stefoni S. Citrate and mineral metabolism: kidney stones and bone metabolism. Front Bioscience 2003;8:s1084-1106.

2. Kaufman AM, Kahn T. Complementary role of citrate and bicarbonate excretion in acid-base balance in the rat. Am J Physiol 1988;255:F182-F187.
3. Simpson DP. Citrate excretion: a window on renal metabolism. Am J Physiol. 1983;244:F223-F234.

4. Goraya N, Simoni J, Sager LN, et al. Urine citrate excretion as a marker of acid retention in patients with chronic kidney disease without overt metabolic acidosis. Kidney Int 2019;95:1190-1196.

5. Brown JC, Packer RK, Knepper MA. Role of organic anions in renal response to dietary acid and base loads. Am J Physiol. 1989;257:F170-F176.

6. Costello LC, Franklin RB. Plasma citrate homeostasis: How it is regulated; and its physiological and clinical implications. An important, but neglected, relationship in medicine. HSOA J Hum Endocrinol 2016;1;pii: 005.

7. Kang $\mathrm{DE}$, Sur RL, Haleblian GE, et al. Long-term lemonade based dietary manipulation in patients with hypocitraturic nephrolithiasis. J Urol 2007;177:1358-1362.

8. Ivanchenko $P$, Delgado-López JM, lafisco $M$, et al. On the surface effects of citrates on nano-apatites: evidence of a decreased hydrophilicity. Sci Rep 2017;7:8901.

9. Costello LC, Chellaiah M, Zou J, Franklin RB, Reynolds MA. The status of citrate in the hydroxyapatite/collagen complex of bone; and Its role in bone formation. J Regen Med Tissue Eng 2015;3:4.

10. Kenny A, Draskczy P, Goldhaber P. Citric acid production by resorbing bone in tissue culture. Am J Physiol. 1959;197:502-504.

11. Dixon TF, Perkins HR. Citric acid and bone metabolism. Biochem J. 1952;52:260-265.

12. Fu X, Li $\mathrm{Y}$, Huang $\mathrm{T}$, et al. Runx2/Osterix and zinc uptake synergize to orchestrate osteogenic differentiation and citrate containing bone apatite formation. Adv Sci 2018;5:1700755.

13 Chen $\mathrm{H}$, Wang $\mathrm{Y}$, Dai $\mathrm{H}$, et al. Bone and plasma citrate is reduced in osteoporosis. Bone 2018;114:189-197.

14. Herzberg M, Foldes J, Steinberg R, Menczel J. Zinc excretion in osteoporotic women. J Bone Miner Res 1990;5:251-257.

15. M. Yamaguchi, M. Goto, S. Uchiyama, T. Nakagawa. Effect of zinc on gene expression in osteoblastic MC3T3-E1 cells: enhancement of Runx2, OPG, and regucalcin mRNA expressions. Mol Cell Biochem 2008;312:157-166.

16. Hu YY, Rawal A, Schmidt-Rohr K. Strongly bound citrate stabilizes the apatite nanocrystals in bone. Proc Natl Acad Sci USA 2010;107:22425-22429.

17. Davies E, Müller KH, Wong WC. Citrate bridges between mineral platelets in bone. Proc Natl Acad Sci USA 2014;111: E1354-E1363.

18. Ivanchenko P, Delgado-López JM, lafisco $M$, et al. On the surface effects of citrates on nano-apatites: evidence of a decreased hydrophilicity. Sci Rep 2017;7:8901.

19. Irizarry AR, Yan G, Zeng Q. Defective enamel and bone development in sodium-dependent citrate transporter (NaCT) Slc13a5 deficient mice. PLoS ONE 2017;12:e0175465.

20. Caudarella R, Miniero R, Rizzoli E, et al. Urinary citrate excretion in healthy women before and after menopause. Ital J Mineral Electrolyte Metab 1995;9:31-38.

21. Jehle S, Hulter HN, Krapf R. Effect of potassium citrate on bone density, microarchitecture, and fracture risk in healthy older adults without osteoporosis: a randomized controlled trial. J Clin Endocrinol Metab 2013;98:207-217.

22. Vescini F, Buffa A, La Manna G, et al. Long-term potassium citrate therapy and bone mineral density in idiopathic calcium stone formers. J Endocrinol Invest 2005;28:218-222.

23. Jehle S, Zanetti A, Muser J, et al. Partial neutralization of the acidogenic western diet with potassium citrate increases bone mass in postmenopausal women with osteopenia. J Am Soc Nephrol 2006;17:3213-3222.

24. Marangella M, Di Stefano M, Casalis S, Berutti S, D'Amelio P, Isaia GC. Effects of potassium citrate supplementation on bone metabolism. Calcif Tissue Int 2004;74:330-335. 
25. Gregory NS, Kumar R, Stein EM, Alexander E, Christos P, Bockman RS, Rodman JS. Potassium citrate decreases bone resorption in postmenopausal women with osteopenia: A randomized double-blind clinical trial. Endocr Pract 2015;21:1380-1386.

26. Sebastian A, Harris ST, Ottaway JH, et al. Improved mineral balance and skeletal metabolism in postmenopausal women treated with potassium bicarbonate. N Engl J Med 1994;330: 1776-1781.

27. Nielsen TT, Sorensen NS. Citrate in plasma and urine during total fasting. Acta Med Scand 1979;205:303-307.

28. Marangella $M$, Vitale $C$, Manganaro $M$, et al. Renal handling of citrate in chronic renal insufficiency. Nephron 1991;57:439-443.

29. Fraenkl SA, Muser J, Groell R, et al. Plasma citrate levels as a potential biomarker for glaucoma. J Ocul Pharmacol Ther 2011;27:577-580.
30. Tiselius HG, Berg C, Fornander AM, Nilsson MA. Effects of citrate on the different phases of calcium oxalate crystallization. Scanning Microsc 1993;7:381-390.

31. Phillips R, Hanchanale VS, Myatt A, Somani B, Nabi G, Biyani CS. Citrate salts for preventing and treating calcium containing kidney stones in adults. Cochrane Database Syst Rev 2015; (10):CD010057.

32. Granchi D, Torreggiani E, Massa A, Caudarella R, Di Pompo G, Baldini N. Potassium citrate prevents increased osteoclastogenesis resulting from acidic conditions: Implication for the treatment of post-menopausal bone loss. PLoS One 2017;12: e0181230. 